\title{
Etiquetas diagnósticas más \\ frecuentes en personas con lesión \\ medular en fase de rehabilitación
}

\section{Nursing diagnosis tags most frequently performed for spinal cord injury people in rehabilitation phase}

Mtra: Sandra Hernández Corral* Lic. Adolfo Acosta Ángeles**

*Jefe de Servicio Instituto Nacional de Rehabilitación EER, ** Jefe de Servicio Instituto Nacional de Rehabilitación

\section{Resumen}

Los diagnósticos de Enfermería desde el punto de vista de la gestión del cuidado pueden ser utilizados como criterios clave conceptuales alrededor de los cuales se desarrollan estándares, como es el caso de los planes de cuidado estandarizado.

La finalidad del estudio fue Identificar las etiquetas diagnósticas de Enfermería más frecuentes en personas con LM en fase de rehabilitación en el servicio de Lesionados Medulares.

Metodología: estudio descriptivo, observacional, transversal; muestra: estuvo integrada por 90 registros realizados por las enfermeras del servicio. La recolección de datos se realizó a través de un instrumento para identificar las etiquetas diagnósticas más frecuentes de la persona con lesión medular de acuerdo a la Taxonomía de la Asociación Norteamericana de

The purpose of this study was to identify the most frequently nursing diagnosis tags in people with spinal Cord Injury (SCI) in rehabilitation process at Spinal cord Injured Ward.

Methodology, this is a descriptive, obsenvational, transversal; simple: integrated by 90 clinical nursing records performed by nursing staff. The data collection was performed through a specific instrument in order to identify the most frequently nursing diagnosis tags for the $\mathrm{SCl}$ people according to the North American Nursing Diagnosis Association Taxonomy (20052006). Data were analyzed with descriptive statistical techniques.
Diagnósticos de Enfermería (2005-2006). Para el análisis se utilizó estadística descriptiva.

Los resultados obtenidos fueron: deterioro de la movilidad física, riesgo de deterioro de la integridad cutánea, déficit de autocuidado: baño, vestido, deterioro de la integridad cutánea, estreñimiento, incontinencia urinaria refleja, incontinencia fecal, limpieza ineficaz de las vías aéreas, disrreflexia autonómica, deterioro de la habilidad para la traslación y otros. Con base en los resultados obtenidos se identificarán las intervenciones de Enfermería y los resultados esperados a pacientes con lesión medular.

Palabras clave: lesión medular, diagnósticos de Enfermería, plan de cuidados.

\section{Abstract}

The results were: physical movement impairment, skin integrity damage risk, self-care deficit: bathing, dressing; skin integrity damage, constipation, reflex urinary tract incontinence, fecal incontinence, ineffective clean up of the airways, autonomic dysreflexia, impairment of the ability to pass and others diagnosis. Based on the results, the Nursing Interventions and the Nursing Outcomes will be identified specifically for SCI patients.

Key words: spinal cord injury, nursing diagnosis, nursing care plan 


\section{INTRODUCCIÓN}

El diagnóstico de Enfermería es considerado como la segunda etapa del proceso de Enfermería, que consiste en el establecimiento de conclusiones relativas a los datos que se han recolectado y analizado. Por tanto el diagnóstico permitirá, mediante un término concreto, entender la situación de la persona, en la que se identifican tanto aspectos positivos como negativos. Se debe entender el diagnóstico, de forma general, como un juicio acerca de la problemática de la persona, al que se llega mediante procesos deductivos a partir de los datos recolectados. La índole del problema es tal que puede resolverse mediante la intervención enfermera.

Es importante resaltar la existencia de varias formas de expresar los diagnósticos de Enfermería. El sistema más utilizado fue desarrollado por la Asociación Norteamericana de Diagnósticos de Enfermería (North American Nursing Diagnosis Asociattion (NANDA).

Entre los propósitos para utilizar diagnósticos de Enfermería se pueden considera los siguientes: Contribuyen a la identidad de la Enfermería como profesión, proporcionan un mecanismo de expresión profesional, facilitan la autonomía de las enfermeras en la emisión de juicios acerca del cuidado de la persona y contribuyen a la continuidad del cuidado.

Algunos autores enfocan su atención en las ventajas de los diagnósticos de Enfermería desde el punto de vista de la gestión del cuidado entre las que se encuentra el utilizarlos como "criterios clave conceptuales alrededor de los cuales se desarrollan estándares", ${ }^{2}$ como es el caso de los planes de cuidado estandarizado, en los cuales los diagnósticos de Enfermería son considerados como prevalentes. ${ }^{3}$

En este sentido, la situación actual con un enfoque de economía de la salud obliga a las instituciones de salud a establecer un control más racional de los recursos. Se asume desde las administraciones que el costo ha de estar basado en la evidencia, lo cual en la profesión de Enfermería se suele traducir a la medición de la tarea derivada de la actuación médica o en algunos casos por los grados de dependencia del paciente, ya que el uso de escalas no es suficiente para reflejar la realidad de las necesidades y demandas del usuario, ni el trabajo enfermero.

Se pretende que, cada intervención registrada en un plan de cuidados estandarizado pueda permitir el cálculo de la carga de trabajo de los cuidados, teniendo en cuenta las intervenciones seleccionadas para la persona.

En México existen pocos servicios en el ámbito público, que ofrecen rehabilitación hospitalaria a los pacientes con
Lesión Medular (LM); actualmente el Instituto Nacional de Rehabilitación es uno de ellos. El hecho de proporcionar cuidado de alta especialidad demanda del personal de Enfermería que labora en él una actualización en el manejo de dichos pacientes, situación que originó la necesidad de crear estrategias de capacitación continua, por lo que se consideró el identificar las etiquetas diagnósticas más frecuentes en pacientes con lesión medular en fase de rehabilitación, teniendo como precedente que el personal de Enfermería de rehabilitación de manera cotidiana elabora diagnósticos de Enfermería en todos las personas hospitalizados en esta área.

Para la identificación de los diagnósticos de Enfermería más frecuentes se realizó una revisión previa de las características generales que presentan las personas con lesión medular ingresados al servicio para su rehabilitación, reconociendo diversas problemáticas, siendo las más frecuentes: problemas del tracto urinario, estos pueden originar una pérdida en el control vesical; problemas Intestinales, generalmente existe alteración en el patrón de vaciamiento que va del estreñimiento a la incontinencia fecal; úlceras por

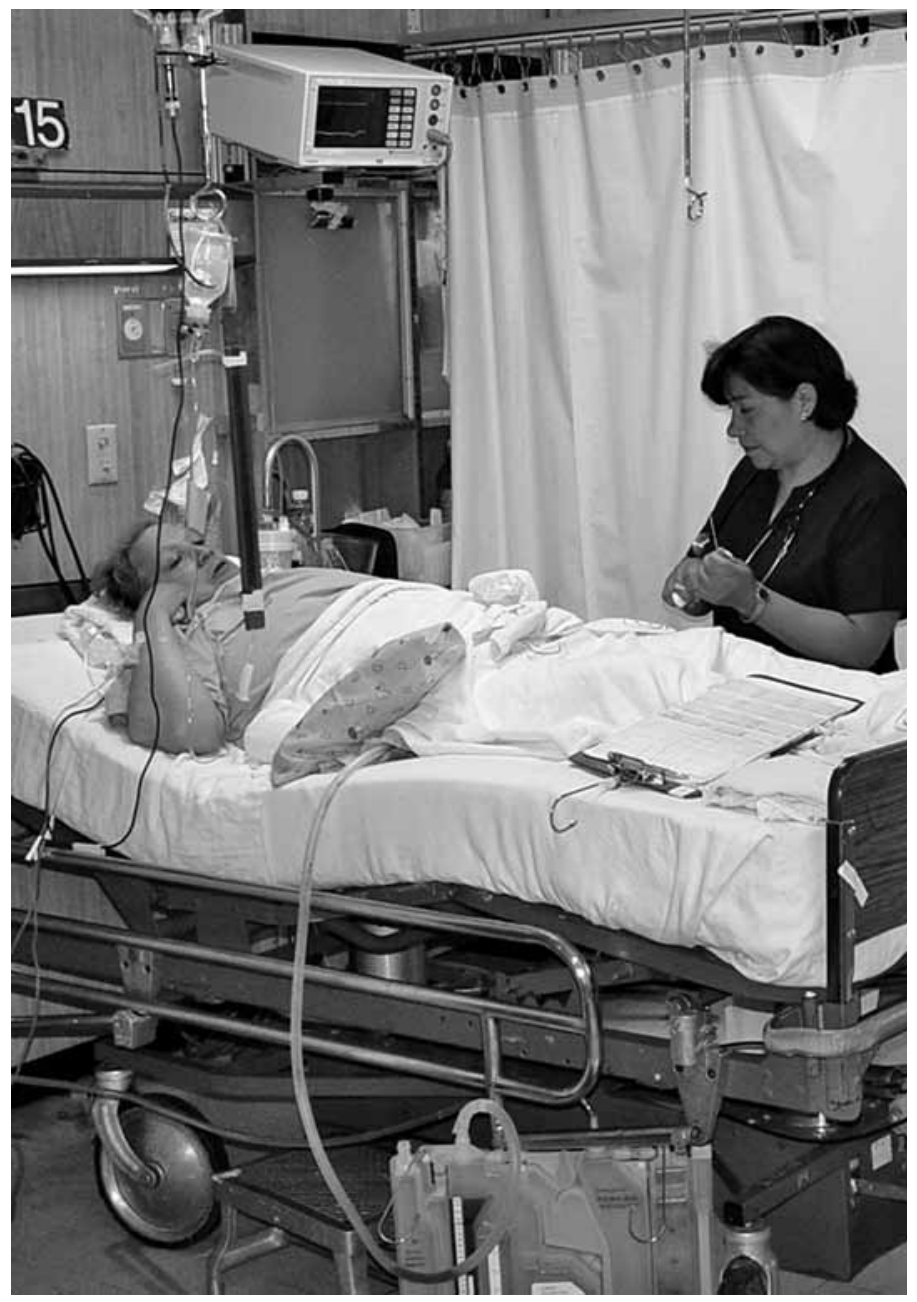


presión, causadas por la pérdida de la sensibilidad y movilidad volitiva, total o parcial; trombosis venosa profunda y embolismo pulmonar, ocasionado por reposo prolongado; problemas ventilatorios y pulmonares, a las personas con LM les resulta más difícil el ventilar y toser con unos músculos abdominales y torácicos débiles, de tal manera que con una LM cervical o torácica se puede desarrollar neumonía u otros problemas pulmonares; disrreflexia autonómica, se presenta en lesionados medulares a nivel neurológico de T6 o por arriba, aunque también se presenta en personas con un nivel de lesión más bajo. Esta condición se caracteriza por un incremento en la tensión arterial, suele presentase de manera refleja, súbita y exagerada, algunas veces acompañada de bradicardia, como respuesta a un estimulo originado por debajo del nivel de lesión; espasticidad, entendida como un estado de actividad refleja excesiva asociada con movimientos involuntarios, clonus y la presencia de reflejos primitivos pone en riesgo a la persona de desarrollar contracturas debilitantes que interfieren con la movilidad; dolor, está en función del daño que sufre la médula espinal o de otras partes del cuerpo, es posible que se experimente en áreas del cuerpo donde la sensación esta afectada, de igual manera se puede presentar por sobreuso muscular. Cualquier clase de dolor impacta de manera negativa en las actividades de la vida diaria. Finalmente, el paciente con LM es susceptible a desarrollar lesiones nuevas en cualquier parte del cuerpo secundarias a la lesión misma, pueden incluso quemarse o cortarse sin tomar conciencia de ello., 5, 6,7

\section{METODOLOGÍA}

Se realizó un estudio descriptivo, observacional y transversal, durante los meses de febrero a noviembre del 2007, en el servicio de Lesionados Medulares del INR.

La muestra fue no probabilística, por conveniencia, seleccionando 90 registros de Enfermería de los pacientes con diagnóstico de LM independientemente del nivel neurológico de lesión, que se encontraban hospitalizados en las fechas en que se realizó el estudio.

Los diagnósticos de Enfermería fueron elaborados por: Enfermeras generales (16.7\%), Enfermeras especialistas (30\%) y Licenciados en Enfermería (53.7\%), de los tres turnos.

La recolección de datos se realizó a través de un Inventario de los diagnósticos enfermeros más frecuentes en personas con Lesión medular teniendo como sustento la Taxonomía de la Asociación Norteamericana de Diagnósticos de Enfermería (NANDA 2005-2006). ${ }^{8}$
Los datos se procesaron con estadística descriptiva. Desde el punto de vista ético, la investigación es de riesgo mínimo.

\section{RESULTADOS}

La antigüedad laboral del personal de Enfermería que realizó los registros fue: menos de un año (33.3\%), entre 1 y 4 años (50\%) y más de 5 años (16.7\%). Referente a la información recolectada según el tuno se tiene: matutino (33.3\%), vespertino (16.7\%) y nocturno (50\%).

Las etiquetas diagnósticas de Enfermería más frecuentes fueron las siguientes: Deterioro de la movilidad física y Riesgo de deterioro de la integridad cutánea (100\%); Déficit de autocuidado: baño (90\%); Déficit de autocuidado: vestido (88\%); Deterioro de la integridad cutánea (85\%); Estreñimiento (83\%); Incontinencia urinaria refleja (80\%), Incontinencia fecal (70\%); Limpieza ineficaz de las vías aéreas (60\%), Disrreflexia autonómica (58\%); Deterioro de la habilidad para la traslación (50\%). Otros diagnósticos que se presentaron con menor frecuencia son: Ansiedad, Hipertermia, Perfusión tisular periférica ineficaz, Duelo y Déficit de conocimientos (10\%).

\section{DISCUSIÓN}

El diagnóstico de Enfermería dentro del expediente formaliza la realización de los cuidados y en particular de los factores relacionados con el diagnóstico, contribuyendo al seguimiento y a la continuidad de los cuidados.

En las personas con lesión medular el objetivo de los cuidados de Enfermería de rehabilitación están encaminados a reducir las secuelas debidas a la lesión para alcanzar la mayor independencia posible, prevenir complicaciones y fomentar el autocuidado. ${ }^{9}$

En este sentido el autocuidado en la persona con lesión medular tiene como finalidad mejorar la calidad de vida, además de aportar beneficios de dos tipos:

Una aceptación activa y equilibrada de la enfermedad, que dará paso a una autorregulación y autocontrol de la enfermedad y una prevención hacia los factores de riesgo que afectarían su calidad de vida.

El fomento del autocuidado debe iniciarse desde la fase aguda de la lesión, en ésta, se enseña al paciente y familiar los cuidados preventivos, para evitar complicaciones como son: infecciones de vías urinarias, úlceras por presión, infecciones respiratorias, hipotensión ortostática, disrreflexia, entre otras. ${ }^{10,11}$ En este sentido, las etiquetas diagnósticas más frecuentemente registradas fueron: Deterioro de la mo- 
vilidad física, Riesgo de deterioro de la integridad cutánea, Deterioro de la integridad cutánea, Estreñimiento, Incontinencia urinaria refleja, Incontinencia fecal, Limpieza ineficaz de las vías aéreas, Disrreflexia.

\section{CONCLUSIONES}

El identificar las etiquetas diagnósticas más frecuentes en personas con LM en fase de rehabilitación permitirá identifi-

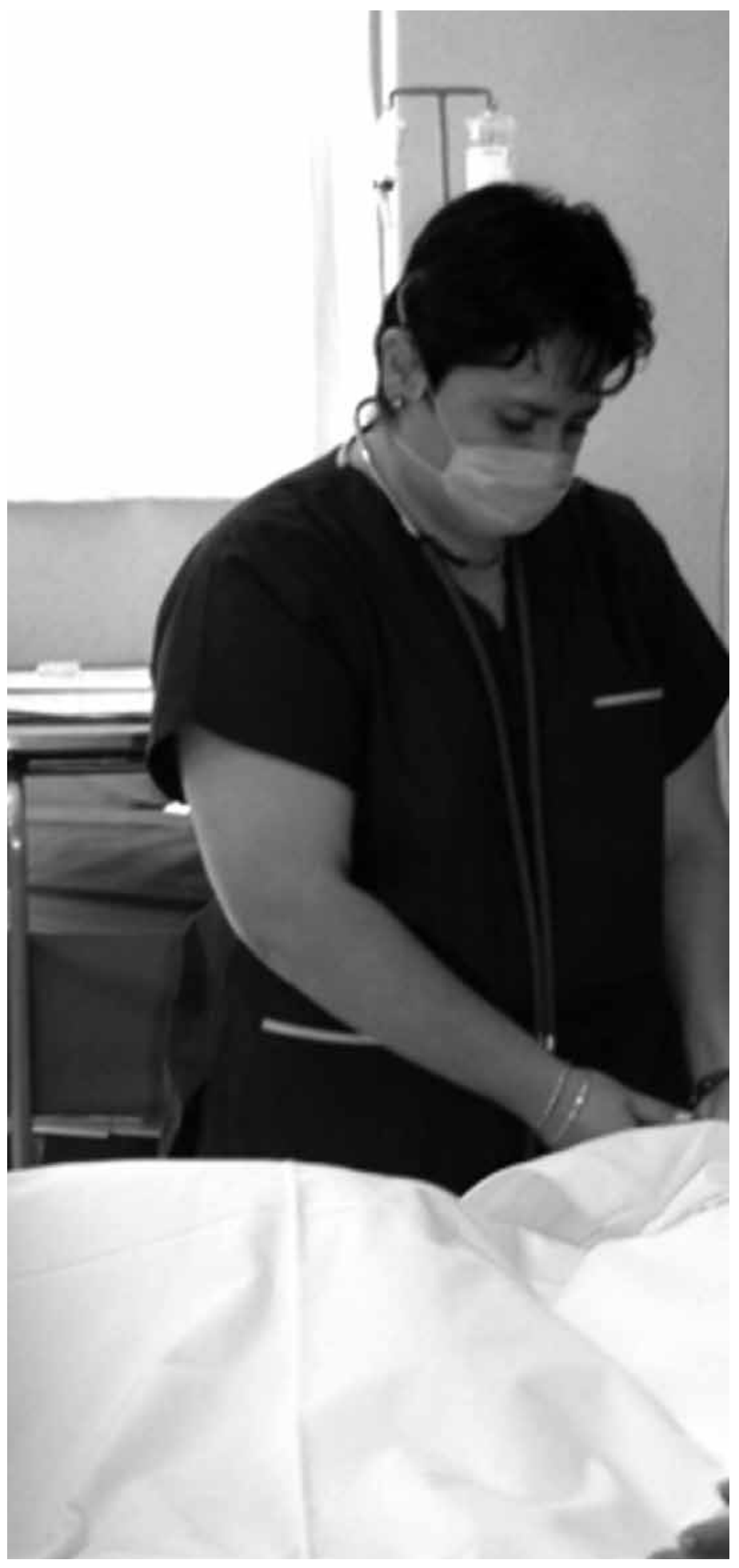

car las intervenciones y los resultados como parte de un plan de cuidados estandarizados en este tipo de pacientes.

\section{REFERENCIAS BIBLIOGRÁFICAS}

1 Hernández CJ, Esteban AM. El método de Intervención en Enfermería: El Proceso de Enfermería. En: Hernández CJ, Esteban AM, editores. Fundamentos de Enfermería. Teoría y Método. España: McGraw-Hill Interamericana; 1999. p. 127-140

2 Baker R. Diagnosis de Enfermería: Declaraciones de Diagnóstico. En: Christensen P, Kenney J. Editors. Nursing Process: Application of conceptual Models. USA;1990.

3 Charrier J, Ritter B. El plan de cuidados estandarizado. Un soporte del diagnóstico enfermero. Elaboración y propuesta. Barcelona: Masson; 2003. p. 4, 22-33

4 Campagnolo D. Autonomic Dysreflexia. Spinal Cord Injury. Oct. 2006. (consultado 29/05/08 on line). Disponible en: www.emedicine.com/pmr/TOPIC217.HTM

5 McKinney D. Prevention of Thromboembolism. Spinal Cord Injury. Mar 2006. (consultado 29/05/08 on line). Disponible en: Disponible en: www.emedicine.com/pmr/TOPIC229.HTM

6 McKinley W. Cardiovascular Concerns. Spinal Cord Injury. Feb. 2008. (consultado 25/06/08 on line). Disponible en:: www.emedicine.com/pmr/TOPIC20.HTM

7 Merrit JL. Management of spasticity. Spinal cord injury. Mayo Clin Proc, 1981; 56: 614-622

8 Salzberg A. Byrne D, Kabir R Predicting Pressure Ulcers During Initial Hospitalization for Acute Spinal Cord Injury. Wounds 1999; 11(2):45-57

9 Asociación Norteamericana de Diagnósticos de Enfermería. Diagnósticos enfermeros: definición y clasificación 20052006. Madrid: Elsevier; 2005. 150p.

10 García RL, Giraldo MC. El que hacer de la enfermera especialista en rehabilitación en un hospital de tercer nivel de atención. Investigación y educación en Enfermería. (consultado 15 de mayo 2008 on line ) Disponible en: http:// www.tone.udea.edu.co/revista/sep95

11 Mancussi FA. A reabilitaçao da pessoa com lesao medular: tendencias da investigaçao no Brasil. Enfermería Global (consultado 5 de mayo 2008 on line ) Disponible en: http:// ww.um.es/global/2003

\section{DIRECCIÓN PARA CORRESPONDENCIA}

Sandra Hernández Corral: hecs720915@yahoo.com.mx Lic. Adolfo Acosta Ángeles: ariesone74@yahoo.com 